Canadian

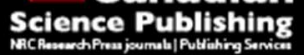

Canadian Journal of Physics

Revue canadienne de physique

\title{
The Pioneer Anomaly in Covariant Theory of Gravitation
}

\begin{tabular}{|c|c|}
\hline Journal: & Canadian Journal of Physics \\
\hline Manuscript ID: & cjp-2015-0134.R1 \\
\hline Manuscript Type: & Article \\
\hline Date Submitted by the Author: & 25-Apr-2015 \\
\hline Complete List of Authors: & Fedosin, Sergey \\
\hline Keyword: & $\begin{array}{l}\text { Pioneer anomaly, covariant theory of gravitation, general theory of } \\
\text { relativity, equation of motion, flyby anomaly }\end{array}$ \\
\hline
\end{tabular}

SCHOLARONE ${ }^{\text {IM }}$

Manuscripts 


\title{
The Pioneer Anomaly in Covariant Theory of Gravitation
}

\author{
Sergey G. Fedosin \\ Sviazeva Str. 22-79, Perm, 614088, Perm region, Russian Federation \\ Phone+79129870408, e-mail $\underline{\text { intelli@list.ru }}$
}

\begin{abstract}
The difference of equations of motion in the covariant theory of gravitation and in the general theory of relativity is used for explanation of Pioneer anomaly. Calculation shows that the velocities of a spacecraft in both theories at equal distances can differ by several $\mathrm{cm} / \mathrm{s}$. This leads also to possible explanation of flyby anomaly and disturbances of comet which are not taken into account by the general theory of relativity.

Keywords: Pioneer anomaly, covariant theory of gravitation, general theory of relativity, equation of motion, flyby anomaly.

PACS: 04.50.Kd, 04.20.Jb, 04.80.-y, 95.55.Pe
\end{abstract}

Résumé: Les differences entre les équations de mouvement dans la théorie covariante de la gravitation et dans la théorie générale de la gravitation sont utilisées pour expliquer "L'anomalie Pioneer". Le calcul montre que les vélocités des sondes spatiales dans les deux theories aux distances différentes peuvent se différencier à plusieurs $\mathrm{cm} / \mathrm{s}$. Cela amène à l'explication possible de l'anomalie "flyby" et aux perturbations de la comète qui ne sont pas pris en compte dans la théorie générale de la relativité.

\section{Introduction}

The story of the American spacecrafts Pioneer 10 and Pioneer 11 began on March 2, 1972, and, respectively, on April 6, 1973, at the time of their launch. Both spacecrafts passed in the plane of the ecliptic the entire Solar system in two opposite directions, passing close to different planets. Pioneer 10 on December 4, 1973, reached Jupiter, located at the distance of 
5.2 a.u. from the Sun $\left(1\right.$ a.u. $\left.=1.496 \cdot 10^{11} \mathrm{~m}\right)$, in June 1983 it passed Pluto (39.4 a.u.), in May 2001 it was at the distance of 78 a.u., moving at the speed of nearly $13 \mathrm{~km} / \mathrm{s}$.

Starting from the distance of about 20 a.u., when in the Doppler signal from Pioneer 10 the shift of the speed significantly decreased, caused by the pressure of the solar plasma on the spacecraft, after taking into account all other possible causes of acceleration, the residual signal from the spacecraft started to show the presence of an anomalous acceleration towards the Sun, of the order of $8 \cdot 10^{-10} \mathrm{~m} / \mathrm{s}^{2}$ [1]. For Pioneer 11 the similar acceleration was about $8.6 \cdot 10^{-10} \mathrm{~m} / \mathrm{s}^{2}$, for the spacecraft Ulysses at distances of $1.3-5.2$ a.u. the acceleration reached $(12 \pm 3) \cdot 10^{-10} \mathrm{~m} / \mathrm{s}^{2}$, while for the spacecraft Galileo $-8 \cdot 10^{-10} \mathrm{~m} / \mathrm{s}^{2}$.

There are some possible explanations of anomalous acceleration of the spacecrafts. One of them for the Pioneer 10 and 11 spacecraft is due to the recoil force associated with an anisotropic emission of thermal radiation off the vehicles [2-3]. The other explanations of Pioneer anomaly include new gravitational physical mechanisms [4-10].

The covariant theory of gravitation (CTG) is an alternative theory for general theory of relativity (GTR) and we present further CTG approach to the problem of the Pioneer anomaly by comparing of calculations of CTG and GTR.

\section{Metric tensor in CTG}

The metric tensor in spherical coordinates $x^{0}=c t, x^{1}=r, x^{2}=Q, x^{3}=\varphi$ has the following form:

$$
g_{i k}=\left(\begin{array}{cccc}
B & 0 & 0 & 0 \\
0 & -K & 0 & 0 \\
0 & 0 & -E & 0 \\
0 & 0 & 0 & -E \sin ^{2} Q
\end{array}\right)
$$


and for the functions $B, K, E$ we assume that they are the functions only of the radial coordinate $r$ as the distance from the center of the massive body (where we placed the origin) to the observation point, located outside the body.

The components of the metric tensor $g_{i k}$ are as follows [11-12]:

$$
\begin{aligned}
& B=g_{00}=1+\frac{G M \alpha}{r c^{2}}-\frac{\beta G^{2} M^{2}}{r^{2} c^{4}}, \quad E=-g_{22}=r^{2} \\
& K=-g_{11}=\frac{1}{B}=\frac{1}{1+\frac{G M \alpha}{r c^{2}}-\frac{\beta G^{2} M^{2}}{r^{2} c^{4}}}, \quad g_{33}=-r^{2} \sin ^{2} Q \\
& g^{00}=\frac{1}{B}, \quad g^{11}=-B, \quad g^{22}=-\frac{1}{r^{2}}, \quad g^{33}=-\frac{1}{r^{2} \sin ^{2} Q}
\end{aligned}
$$

where $\alpha$ and $\beta$ are the values which cannot be determined from the equations for the metric, which leads to their possible dependence on the properties of test particles in the gravitational field,

$M$ is the body mass, near which the metric is determined,

$G$ is the gravitational constant,

$c=c_{g}$ is the speed of gravitation propagation.

We shall express the metric tensor $g^{\text {in }}$ in terms of the Cartesian coordinates. For the relation of the Cartesian and the spherical coordinates we have:

$$
x=r \sin Q \cos \varphi, \quad y=r \sin Q \sin \varphi, \quad z=r \cos Q .
$$




$$
x^{2}+y^{2}+z^{2}=r^{2}
$$

$$
d x=d r \sin Q \cos \varphi+d Q r \cos Q \cos \varphi-d \varphi r \sin Q \sin \varphi
$$

$$
d y=d r \sin Q \sin \varphi+d Q r \cos Q \sin \varphi+d \varphi r \sin Q \cos \varphi
$$

$$
\begin{gathered}
d z=d r \cos Q-d Q r \sin Q . \\
(d \ell)^{2}=(d x)^{2}+(d y)^{2}+(d z)^{2}=(d r)^{2}+(r d Q)^{2}+(r \sin Q d \varphi)^{2}, \\
d \vec{\ell}=(d x, d y, d z), \quad d \vec{\ell}=(d r, r d Q, r \sin Q d \varphi) .
\end{gathered}
$$

The relations (3) are the rules with the help of which by the known the spherical coordinates $r, Q, \varphi$ the Cartesian coordinates of the point are found. Due to this definition the equality (4) for the Cartesian coordinates will hold in the Riemannian space.

In (6) the 3 -vector of displacement of the test particle $\overrightarrow{d \ell}$ has projections on three mutually perpendicular axes of the Cartesian coordinate system, equal to $d x, d y$ and $d z$. The similar projections of the 3-vector $\overrightarrow{d \ell}$ on three mutually perpendicular axes of the spherical coordinate system are equal to $d r, r d Q$ and $r \sin Q d \varphi$. One unit vector in the spherical coordinate system is directed along the radial coordinate $r$ and the other two are perpendicular to it and are directed along the meridians and parallels, where the changes of the angles $d Q$ and $d \varphi$ are measured. 
In view of (6) the 4-vector of displacement which is symmetrical with respect to dimensions (the 4-vector of the distance differential) in the spherical coordinates has the form:

$$
d x^{i}=(c d t, d r, r d Q, r \sin Q d \varphi)
$$

To find $g^{\text {in }}$ in the Cartesian coordinates through its form in the spherical coordinates (2) we must take into account the existing relationship between the coordinates and the components of the 4 -vector of displacement. In the Cartesian coordinates $x^{0}=c t, x^{1}=x$, $x^{2}=y, x^{3}=z$, and $d x^{i}=(c d t, d x, d y, d z)$, so to obtain the 4-vector of displacement it is sufficient to take the differentials of the coordinates. For the spherical coordinates $x^{0}=c t$, $x^{1}=r, x^{2}=Q, x^{3}=\varphi$, but to obtain the corresponding 4-vector of displacement is not enough just to use the differentials of the coordinates, we must also multiply them by some functions of the coordinates, as it is seen in (7). Only in this case it becomes possible to compare the 4-vectors of displacement, expressed in different reference frames.

However, as it follows from the metric (2), the various components of the metric tensor in the spherical coordinates, as well as the corresponding Christoffel coefficients have different dimensions. This means that the 4-vector of displacement in the spherical coordinates should be asymmetric with respect to dimension and have the form:

$$
d x^{i}=(c d t, d r, d Q, d \varphi)
$$


In general case, the transformation of 4 -vectors and tensors from one frame to another is performed by using the transformation matrices of the form $A_{k}^{i}=\frac{\partial x^{\prime i}}{\partial x^{k}}$ and $B_{k}{ }^{i}=\frac{\partial x^{i}}{\partial x^{\prime k}}$, so for an arbitrary tensor the transformation of 4-coordinates $x^{i}$ into the 4-coordinates $x^{\prime i}$ is valid:

$$
T_{r s \ldots}^{m n \ldots .}{ }^{\prime}\left(x^{i}\right)=A_{k}^{m} A_{l}^{n} \ldots B_{r}{ }^{p} B_{s}^{q} \ldots T_{p q \ldots}^{k l \ldots}\left(x^{i}\right) .
$$

We shall find the transformation matrix $A_{k}^{i}$ of such kind, that with the help of it the 4vector (8) could be transformed into the 4-vector of displacement in the Cartesian coordinates. If we take into account the relations for the differentials (5), which give the expressions for the corresponding partial derivatives, standing before the differentials, then we shall obtain:

$$
\begin{gathered}
A_{k}^{i}=\left(\begin{array}{cccc}
1 & 0 & 0 & 0 \\
0 & \sin Q \cos \varphi & r \cos Q \cos \varphi & -r \sin Q \sin \varphi \\
0 & \sin Q \sin \varphi & r \cos Q \sin \varphi & r \sin Q \cos \varphi \\
0 & \cos Q & -r \sin Q & 0
\end{array}\right), \\
d x^{i}(c t, x, y, z)=A_{k}^{i} d x^{k}(c t, r, Q, \varphi)
\end{gathered}
$$

where $d x^{k}$ is from (8).

To complete the transition from the spherical to Cartesian variables, the angles $Q$ and $\varphi$ in (10) should be expressed through $x, y$ and $z$ with the help of (3).

Applying to the tensor $g^{i k}$ from (2) the transformation (9) with the help of $A_{k}^{i}$ from (10) we find the corresponding metric tensor in the Cartesian variables: 


$$
g^{m n}(c t, x, y, z)=A_{i}^{m} A_{k}^{n} g^{i k}(c t, r, Q, \varphi)
$$

$$
g^{m n}=\left(\begin{array}{cccc}
\frac{1}{B} & 0 & 0 & 0 \\
0 & C \sin ^{2} Q \cos ^{2} \varphi-1 & C \sin ^{2} Q \sin \varphi \cos \varphi & C \sin Q \cos Q \cos \varphi \\
0 & C \sin ^{2} Q \sin \varphi \cos \varphi & C \sin ^{2} Q \sin ^{2} \varphi-1 & C \sin Q \cos Q \sin \varphi \\
0 & C \sin Q \cos Q \cos \varphi & C \sin Q \cos Q \sin \varphi & C \cos ^{2} Q-1
\end{array}\right)
$$

where $C=-\frac{G M \alpha}{r c^{2}}+\frac{\beta G^{2} M^{2}}{r^{2} c^{4}}=1-B$

After replacing the trigonometric functions of the angles $Q$ and $\varphi$ through $x, y$ and $z$ with the help of (3) the metric tensor (12) in the Cartesian coordinates becomes as follows:

$$
g^{m n}=\left(\begin{array}{cccc}
\frac{1}{B} & 0 & 0 & 0 \\
0 & \frac{C x^{2}}{r^{2}}-1 & \frac{C x y}{r^{2}} & \frac{C x z}{r^{2}} \\
0 & \frac{C y x}{r^{2}} & \frac{C y^{2}}{r^{2}}-1 & \frac{C y z}{r^{2}} \\
0 & \frac{C z x}{r^{2}} & \frac{C z y}{r^{2}} & \frac{C z^{2}}{r^{2}}-1
\end{array}\right) .
$$

Since for the metric tensor the equality holds: $g_{s m} g^{m n}=\delta_{s}{ }^{n}$, where $\delta_{s}{ }^{n}=\left\{\begin{array}{ll}1, & s=n \\ 0, & s \neq n\end{array}\right.$, it allows us to find $g_{s m}$ by the known form $g^{m n}$. In particular, for each component of the metric tensor with covariant indices we can write down:

$$
g_{s m}=\frac{D^{s m}}{g}
$$


where $D^{s m}$ is the algebraic supplement to the components of the metric tensor $g^{m n}$ with contravariant indices, which is the minor of the matrix of the tensor with the corresponding sign,

$g$ is the determinant of the metric tensor $g^{m n}$, in our case $g=-1$.

Using this rule, we find $g_{s m}$ :

$$
g_{s m}=\left(\begin{array}{cccc}
B & 0 & 0 & 0 \\
0 & -1-\frac{C x^{2}}{B r^{2}} & -\frac{C x y}{B r^{2}} & -\frac{C x z}{B r^{2}} \\
0 & -\frac{C y x}{B r^{2}} & -1-\frac{C y^{2}}{B r^{2}} & -\frac{C y z}{B r^{2}} \\
0 & -\frac{C z x}{B r^{2}} & -\frac{C z y}{B r^{2}} & -1-\frac{C z^{2}}{B r^{2}}
\end{array}\right) .
$$

With the components of the metric tensor (13) and (14) we find the non-zero Christoffel symbols for the Cartesian coordinates:

$$
\begin{gathered}
\Gamma_{i k}^{s}=\frac{1}{2} g^{s m}\left(\partial_{i} g_{m k}+\partial_{k} g_{m i}-\partial_{m} g_{i k}\right), \\
\Gamma_{01}^{0}=\Gamma_{10}^{0}=\frac{B^{\prime} x}{2 B r}, \quad \Gamma_{02}^{0}=\Gamma_{20}^{0}=\frac{B^{\prime} y}{2 B r}, \quad \Gamma_{03}^{0}=\Gamma_{30}^{0}=\frac{B^{\prime} z}{2 B r}, \quad \Gamma_{00}^{1}=\frac{B^{\prime} B x}{2 r}, \\
\Gamma_{11}^{1}=\frac{x(1-B)}{r^{2}}+\frac{x^{3} B}{r^{4}}-\frac{x^{3}\left(B^{\prime} r+2 B\right)}{2 B r^{4}}, \quad \Gamma_{12}^{1}=\Gamma_{21}^{1}=\frac{x^{2} y B}{r^{4}}-\frac{x^{2} y\left(B^{\prime} r+2 B\right)}{2 B r^{4}}, \\
\Gamma_{13}^{1}=\Gamma_{31}^{1}=\frac{x^{2} z B}{r^{4}}-\frac{x^{2} z\left(B^{\prime} r+2 B\right)}{2 B r^{4}}, \quad \Gamma_{22}^{1}=\frac{x(1-B)}{r^{2}}+\frac{x y^{2} B}{r^{4}}-\frac{x y^{2}\left(B^{\prime} r+2 B\right)}{2 B r^{4}},
\end{gathered}
$$




$$
\begin{aligned}
& \Gamma_{23}^{1}=\Gamma_{32}^{1}=\frac{x y z B}{r^{4}}-\frac{x y z\left(B^{\prime} r+2 B\right)}{2 B r^{4}}, \Gamma_{33}^{1}=\frac{x(1-B)}{r^{2}}+\frac{x z^{2} B}{r^{4}}-\frac{x z^{2}\left(B^{\prime} r+2 B\right)}{2 B r^{4}}, \\
& \Gamma_{11}^{2}=\frac{y(1-B)}{r^{2}}+\frac{x^{2} y B}{r^{4}}-\frac{x^{2} y\left(B^{\prime} r+2 B\right)}{2 B r^{4}}, \quad \Gamma_{12}^{2}=\Gamma_{21}^{2}=\frac{x y^{2} B}{r^{4}}-\frac{x y^{2}\left(B^{\prime} r+2 B\right)}{2 B r^{4}}, \\
& \Gamma_{13}^{2}=\Gamma_{31}^{2}=\frac{x y z B}{r^{4}}-\frac{x y z\left(B^{\prime} r+2 B\right)}{2 B r^{4}}, \quad \Gamma_{22}^{2}=\frac{y(1-B)}{r^{2}}+\frac{y^{3} B}{r^{4}}-\frac{y^{3}\left(B^{\prime} r+2 B\right)}{2 B r^{4}}, \\
& \Gamma_{23}^{2}=\Gamma_{32}^{2}=\frac{y^{2} z B}{r^{4}}-\frac{y^{2} z\left(B^{\prime} r+2 B\right)}{2 B r^{4}}, \quad \Gamma_{33}^{2}=\frac{y(1-B)}{r^{2}}+\frac{y z^{2} B}{r^{4}}-\frac{y z^{2}\left(B^{\prime} r+2 B\right)}{2 B r^{4}}, \\
& \Gamma_{00}^{2}=\frac{B^{\prime} B y}{2 r}, \quad \Gamma_{00}^{3}=\frac{B^{\prime} B z}{2 r}, \quad \Gamma_{11}^{3}=\frac{z(1-B)}{r^{2}}+\frac{x^{2} z B}{r^{4}}-\frac{x^{2} z\left(B^{\prime} r+2 B\right)}{2 B r^{4}}, \\
& \Gamma_{12}^{3}=\Gamma_{21}^{3}=\frac{x y z B}{r^{4}}-\frac{x y z\left(B^{\prime} r+2 B\right)}{2 B r^{4}}, \quad \Gamma_{13}^{3}=\Gamma_{31}^{3}=\frac{x z^{2} B}{r^{4}}-\frac{x z^{2}\left(B^{\prime} r+2 B\right)}{2 B r^{4}}, \\
& \Gamma_{22}^{3}=\frac{z(1-B)}{r^{2}}+\frac{y^{2} z B}{r^{4}}-\frac{y^{2} z\left(B^{\prime} r+2 B\right)}{2 B r^{4}}, \Gamma_{23}^{3}=\Gamma_{32}^{3}=\frac{y z^{2} B}{r^{4}}-\frac{y z^{2}\left(B^{\prime} r+2 B\right)}{2 B r^{4}}, \\
& \Gamma_{33}^{3}=\frac{z(1-B)}{r^{2}}+\frac{z^{3} B}{r^{4}}-\frac{z^{3}\left(B^{\prime} r+2 B\right)}{2 B r^{4}},
\end{aligned}
$$

here we used the equalities of the type $\frac{\partial B}{\partial x}=\frac{\partial B}{\partial r} \frac{\partial r}{\partial x}=\frac{B^{\prime} x}{r}$, as well as $r^{2}=x^{2}+y^{2}+z^{2}$. 
With the help of (14) and the expression for the 4-vector of displacement $d x^{s}=(c d t, d x, d y, d z)$ we find the square of the interval:

$$
(d s)^{2}=c^{2}(d \tau)^{2}=g_{s m} d x^{s} d x^{m}=B c^{2}(d t)^{2}-\left[(d x)^{2}+(d y)^{2}+(d z)^{2}\right]-\frac{C}{B r^{2}}(x d x+y d y+z d z)^{2} .
$$

The expression (16) for the square of the interval according to [11] coincides with one of the two so-called normal forms for the Cartesian coordinates [13]. We obtained it, without solving the equations for the metric in the Cartesian coordinates, but making recalculation from the metric in the spherical coordinates.

From (16) for the differential of the proper time of test particle near a massive body it follows:

$$
d \tau=d t \sqrt{B-\frac{1}{c^{2}}\left(\frac{d \ell}{d t}\right)^{2}-\frac{1-B}{B c^{2}}\left(\frac{d r}{d t}\right)^{2}}=d t \sqrt{B-\frac{V^{2}}{c^{2}}-\frac{1-B}{B c^{2}}\left(\frac{d r}{d t}\right)^{2}}
$$

where $V=\frac{d \ell}{d t}$ is the total velocity of the test particle,

and in the derivation of (17) we used the relations: $\left[d\left(x^{2}\right)+d\left(y^{2}\right)+d\left(z^{2}\right)\right]^{2}=\left[d\left(r^{2}\right)\right]^{2}=4 r^{2}(d r)^{2}, \quad(d x)^{2}+(d y)^{2}+(d z)^{2}=(d \ell)^{2}$.

\section{Equation of motion in CTG}

In CTG, in contrast to GTR, there is its own equation of motion of test bodies, which changes the results of the calculations. We shall use the equation of motion of test particles in 
the gravitational field in the form deduced from the principle of least action for CTG [11], [14-16]:

$$
\rho_{0} \frac{d u^{i}}{d \tau}+\Gamma_{k s}^{i} u^{k} J^{s}=g^{i n} \Phi_{n k} J^{k}
$$

where $\rho_{0}$ is the mass density in the reference frame associated with the test particle,

$u^{i}$ are the 4-velocity of the test particle,

$J^{i}=\rho_{0} u^{i}$ is the mass 4-current density,

$d \tau$ is the differential of the proper dynamic time of the test particle,

$\Phi_{n k}$ is the tensor of gravitational field,

$\Gamma_{k s}^{i}$ are the Christoffel symbols.

The 4-vector $J^{i}$ in the Cartesian coordinates can be represented as follows:

$$
J^{i}=\rho_{0} \frac{d x^{i}}{d \tau}=\left(\frac{\rho_{0} c d t}{d \tau}, \frac{\rho_{0} d \vec{\ell}}{d \tau}\right)
$$

where $\overrightarrow{d \ell}=(d x, d y, d z)$.

In the static case the 4 -vector of the gravitational potential has the form $D_{i}=\left(\frac{\psi}{c}, 0,0,0\right)$,

where the scalar potential $\psi=-\frac{G M}{r}$. This gives the tensor of gravitational field strengths with the components: 


$$
\Phi_{i k}=\partial_{i} D_{k}-\partial_{k} D_{i}=\left(\begin{array}{cccc}
0 & -\frac{G M x}{c r^{3}} & -\frac{G M y}{c r^{3}} & -\frac{G M z}{c r^{3}} \\
\frac{G M x}{c r^{3}} & 0 & 0 & 0 \\
\frac{G M y}{c r^{3}} & 0 & 0 & 0 \\
\frac{G M z}{c r^{3}} & 0 & 0 & 0
\end{array}\right) \text {. }
$$

Substituting (19) and (20) into the equations of motion (18), taking into account the metric tensor (13) and the non-zero Christoffel symbols (15), with the values of the index $i=0,1,2,3$, we obtain four equations of motion in the Cartesian coordinates:

$$
\begin{gathered}
\rho_{0} \frac{d u^{0}}{d \tau}+\Gamma_{01}^{0} u^{0} J^{1}+\Gamma_{10}^{0} u^{1} J^{0}+\Gamma_{02}^{0} u^{0} J^{2}+\Gamma_{20}^{0} u^{2} J^{0}+\Gamma_{03}^{0} u^{0} J^{3}+\Gamma_{30}^{0} u^{3} J^{0}=g^{00} \Phi_{0 k} J^{k} \\
\rho_{0} \frac{d u^{1}}{d \tau}+\Gamma_{00}^{1} u^{0} J^{0}+\Gamma_{11}^{1} u^{1} J^{1}+\Gamma_{12}^{1} u^{1} J^{2}+\Gamma_{21}^{1} u^{2} J^{1}+\Gamma_{13}^{1} u^{1} J^{3}+\Gamma_{31}^{1} u^{3} J^{1}+ \\
+\Gamma_{22}^{1} u^{2} J^{2}+\Gamma_{23}^{1} u^{2} J^{3}+\Gamma_{32}^{1} u^{3} J^{2}+\Gamma_{33}^{1} u^{3} J^{3}=g^{1 k} \Phi_{k 0} J^{0} \\
\rho_{0} \frac{d u^{2}}{d \tau}+\Gamma_{00}^{2} u^{0} J^{0}+\Gamma_{11}^{2} u^{1} J^{1}+\Gamma_{12}^{2} u^{1} J^{2}+\Gamma_{21}^{2} u^{2} J^{1}+\Gamma_{13}^{2} u^{1} J^{3}+\Gamma_{31}^{2} u^{3} J^{1}+ \\
+\Gamma_{22}^{2} u^{2} J^{2}+\Gamma_{23}^{2} u^{2} J^{3}+\Gamma_{32}^{2} u^{3} J^{2}+\Gamma_{33}^{2} u^{3} J^{3}=g^{2 k} \Phi_{k 0} J^{0} . \\
\rho_{0} \frac{d u^{3}}{d \tau}+\Gamma_{00}^{3} u^{0} J^{0}+\Gamma_{11}^{3} u^{1} J^{1}+\Gamma_{12}^{3} u^{1} J^{2}+\Gamma_{21}^{3} u^{2} J^{1}+\Gamma_{13}^{3} u^{1} J^{3}+\Gamma_{31}^{3} u^{3} J^{1}+ \\
+\Gamma_{22}^{3} u^{2} J^{2}+\Gamma_{23}^{3} u^{2} J^{3}+\Gamma_{32}^{3} u^{3} J^{2}+\Gamma_{33}^{3} u^{3} J^{3}=g^{3 k} \Phi_{k 0} J^{0}
\end{gathered}
$$

here the nonzero terms are indicated, and by the repeated index $k$, with the values $k=1,2,3$ summation is made as usual. 
We shall write down the equations for the motion in time and for the motion along the axis $O X$ in the explicit form:

$$
\begin{gathered}
\rho_{0} \frac{d}{d \tau}\left(\frac{c d t}{d \tau}\right)+\frac{B^{\prime} \rho_{0} c}{B r} \frac{d t}{d \tau}\left(\frac{x d x}{d \tau}+\frac{y d y}{d \tau}+\frac{z d z}{d \tau}\right)=-\frac{G M \rho_{0}}{B c r^{3}}\left(\frac{x d x}{d \tau}+\frac{y d y}{d \tau}+\frac{z d z}{d \tau}\right) \\
\rho_{0} \frac{d}{d \tau}\left(\frac{d x}{d \tau}\right)+\frac{B^{\prime} B \rho_{0} x c^{2}}{2 r}\left(\frac{d t}{d \tau}\right)^{2}+\frac{(1-B) \rho_{0} x}{r^{2}}\left[\left(\frac{d x}{d \tau}\right)^{2}+\left(\frac{d y}{d \tau}\right)^{2}+\left(\frac{d z}{d \tau}\right)^{2}\right]+ \\
+\frac{\rho_{0} x}{4}\left(\frac{B}{r^{4}}-\frac{B^{\prime} r+2 B}{2 B r^{4}}\right)\left[\frac{d\left(x^{2}\right)}{d \tau}+\frac{d\left(y^{2}\right)}{d \tau}+\frac{d\left(z^{2}\right)}{d \tau}\right]^{2}=-\frac{B G M \rho_{0} x}{r^{3}} \frac{d t}{d \tau}
\end{gathered}
$$

We shall further cancel $\rho_{0}$ in the equations (21-22). By putting $x, y, z$ in (21) under the signs of the differentials and further summation, taking into account the equality $x^{2}+y^{2}+z^{2}=r^{2}$, we can transform the equation (21). Then after multiplying all the parts of the equality (21) by $B$ we shall obtain:

$$
B \frac{d}{d \tau}\left(\frac{d t}{d \tau}\right)+B^{\prime} \frac{d t}{d \tau} \frac{d r}{d \tau}=\frac{d}{d \tau}\left(B \frac{d t}{d \tau}\right)=-\frac{G M}{c^{2} r^{2}} \frac{d r}{d \tau}
$$

here we have used equality $\frac{d B}{d \tau}=\frac{d B}{d r} \frac{d r}{d \tau}=B^{\prime} \frac{d r}{d \tau}$.

The equation (22), taking into account the relations:

$$
\left[d\left(x^{2}\right)+d\left(y^{2}\right)+d\left(z^{2}\right)\right]^{2}=\left[d\left(r^{2}\right)\right]^{2}=4 r^{2}(d r)^{2}, \quad(d x)^{2}+(d y)^{2}+(d z)^{2}=(d \ell)^{2},
$$


can be transformed to the following form:

$$
\frac{d}{d \tau}\left(\frac{d x}{d \tau}\right)+\frac{B^{\prime} B x c^{2}}{2 r}\left(\frac{d t}{d \tau}\right)^{2}+\frac{(1-B) x}{r^{2}}\left(\frac{d \ell}{d \tau}\right)^{2}+x\left(\frac{B}{r^{2}}-\frac{B^{\prime} r+2 B}{2 B r^{2}}\right)\left(\frac{d r}{d \tau}\right)^{2}=-\frac{B G M x}{r^{3}} \frac{d t}{d \tau}
$$

For the motion along the axes $O Y$ and $O Z$, respectively, we obtain:

$$
\begin{aligned}
& \frac{d}{d \tau}\left(\frac{d y}{d \tau}\right)+\frac{B^{\prime} B y c^{2}}{2 r}\left(\frac{d t}{d \tau}\right)^{2}+\frac{(1-B) y}{r^{2}}\left(\frac{d \ell}{d \tau}\right)^{2}+y\left(\frac{B}{r^{2}}-\frac{B^{\prime} r+2 B}{2 B r^{2}}\right)\left(\frac{d r}{d \tau}\right)^{2}=-\frac{B G M y}{r^{3}} \frac{d t}{d \tau} \\
& \frac{d}{d \tau}\left(\frac{d z}{d \tau}\right)+\frac{B^{\prime} B z c^{2}}{2 r}\left(\frac{d t}{d \tau}\right)^{2}+\frac{(1-B) z}{r^{2}}\left(\frac{d \ell}{d \tau}\right)^{2}+z\left(\frac{B}{r^{2}}-\frac{B^{\prime} r+2 B}{2 B r^{2}}\right)\left(\frac{d r}{d \tau}\right)^{2}=-\frac{B G M z}{r^{3}} \frac{d t}{d \tau}
\end{aligned}
$$

In (24) - (26) the value $\frac{d \ell}{d \tau}$ is the total velocity and $\frac{d r}{d \tau}$ is the radial velocity of the test particle. Further we shall consider the case of motion of a test body near the Sun, when the orbit is in the equatorial plane of the spherical coordinate system, and correspondingly in the plane $X O Y$ of the Cartesian coordinate system. Then for the test body $z=0$, the velocity $\frac{d z}{d \tau}=0$, in $(26) \frac{d}{d \tau}\left(\frac{d z}{d \tau}\right)=0$, and over time the coordinate $z$ does not change.

After cancelling $d \tau$ the equation (23) can be integrated:

$$
B \frac{d t}{d \tau}=\frac{G M}{c^{2} r}+A_{1}
$$


At infinity the gravitational influence of the Sun can be neglected, and we can assume that the test body moves inertially. Then the coordinate time $t$ differs from the proper time of the test body $\tau$ only by the Lorentz factor, so we can determine the value of the constant: $A_{1}=\left(\frac{d t}{d \tau}\right)_{\infty}=\frac{1}{\sqrt{1-V_{0}^{2} / c^{2}}}$, where $V_{0}$ is the velocity of the test body at infinity. We can also specify that the velocity $V_{0}$ at infinity must be at least to a small degree being directed to the Sun, otherwise the test body will never get close to it.

To simplify the further solution we shall move in the equations (27), (24), (25) to the polar coordinates in the plane of motion of the test body $X O Y$, in the center of this coordinate system there is the Sun. Substituting $x=r \cos \varphi$ and $y=r \sin \varphi$ in (24) and (25), expressing the total velocity in terms of the radial and tangential velocity components in the form: $\left(\frac{d \ell}{d \tau}\right)^{2}=\left(\frac{d r}{d \tau}\right)^{2}+r^{2}\left(\frac{d \varphi}{d \tau}\right)^{2}$, we find:

$$
\begin{aligned}
&-2 \sin \varphi \frac{d \varphi}{d \tau} \frac{d r}{d \tau}+\cos \varphi \frac{d^{2} r}{d \tau^{2}}-r \sin \varphi \frac{d^{2} \varphi}{d \tau^{2}}+\frac{B^{\prime} B c^{2} \cos \varphi}{2}\left(\frac{d t}{d \tau}\right)^{2}- \\
&- \frac{B^{\prime} \cos \varphi}{2 B}\left(\frac{d r}{d \tau}\right)^{2}-B r \cos \varphi\left(\frac{d \varphi}{d \tau}\right)^{2}=-\frac{B G M \cos \varphi}{r^{2}} \frac{d t}{d \tau} \\
& 2 \cos \varphi \frac{d \varphi}{d \tau} \frac{d r}{d \tau}+\sin \varphi \frac{d^{2} r}{d \tau^{2}}+r \cos \varphi \frac{d^{2} \varphi}{d \tau^{2}}+\frac{B^{\prime} B c^{2} \sin \varphi}{2}\left(\frac{d t}{d \tau}\right)^{2}- \\
&-\frac{B^{\prime} \sin \varphi}{2 B}\left(\frac{d r}{d \tau}\right)^{2}-B r \sin \varphi\left(\frac{d \varphi}{d \tau}\right)^{2}=-\frac{B G M \sin \varphi}{r^{2}} \frac{d t}{d \tau}
\end{aligned}
$$


We can get rid of sines and cosines, if we multiply (28) by $\cos \varphi$ and (29) by $\sin \varphi$, and then, respectively, add the two equations. We can also multiply (28) by $\sin \varphi$ and (29) by $\cos \varphi$ and subtract the equations from each other. The results will be as follows:

$$
\begin{gathered}
\frac{d^{2} r}{d \tau^{2}}+\frac{B^{\prime} B c^{2}}{2}\left(\frac{d t}{d \tau}\right)^{2}-\frac{B^{\prime}}{2 B}\left(\frac{d r}{d \tau}\right)^{2}-B r\left(\frac{d \varphi}{d \tau}\right)^{2}=-\frac{B G M}{r^{2}} \frac{d t}{d \tau} \\
2 \frac{d \varphi}{d \tau} \frac{d r}{d \tau}+r \frac{d^{2} \varphi}{d \tau^{2}}=0
\end{gathered}
$$

The equation (31) is immediately integrated:

$$
\frac{r^{2} d \varphi}{d \tau}=L=\text { const }
$$

From (32) we see that during the motion of the test body the quantity $L$ is preserved, which is proportional to the density of the orbital angular momentum. Dividing (32) by (27), we find:

$$
\frac{d \varphi}{d t}=\frac{B L}{r^{2}\left(\frac{G M}{c^{2} r}+A_{1}\right)} .
$$

Since the square of the total velocity $V$ of the test body in the polar coordinates is composed of the square of the radial component $\dot{r}=\frac{d r}{d t}$ and the square of the tangential 
component $V_{\tau}=r \frac{d \varphi}{d t}$ in the form: $V^{2}=\dot{r}^{2}+V_{\tau}^{2}$, then the differential of the proper dynamic time (17), taking into account (33) will equal:

$$
d \tau=d t \sqrt{B-\frac{\dot{r}^{2}}{B c^{2}}-\frac{B^{2} L^{2}}{r^{2} c^{2}\left(\frac{G M}{c^{2} r}+A_{1}\right)^{2}}}
$$

From (34) and (27) we find $\dot{r}=\frac{d r}{d t}$ and then $\frac{d r}{d \tau}$ :

$$
\begin{gathered}
\frac{d r}{d t}= \pm B \sqrt{c^{2}-\frac{B L^{2}}{r^{2}\left(\frac{G M}{c^{2} r}+A_{1}\right)^{2}}-\frac{B c^{2}}{\left(\frac{G M}{c^{2} r}+A_{1}\right)^{2}}} \\
\frac{d r}{d \tau}= \pm \sqrt{c^{2}\left(\frac{G M}{c^{2} r}+A_{1}\right)^{2}-\frac{B L^{2}}{r^{2}}-B c^{2}}
\end{gathered}
$$

After using (32) in (30) we obtain:

$$
\frac{d^{2} r}{d \tau^{2}}+\frac{B^{\prime} B c^{2}}{2}\left(\frac{d t}{d \tau}\right)^{2}-\frac{B^{\prime}}{2 B}\left(\frac{d r}{d \tau}\right)^{2}-\frac{B L^{2}}{r^{3}}=-\frac{B G M}{r^{2}} \frac{d t}{d \tau}
$$

We shall substitute $\frac{d t}{d \tau}$ from (27) in (37):

$$
\frac{d^{2} r}{d \tau^{2}}+\frac{B^{\prime} c^{2}}{2 B}\left(\frac{G M}{c^{2} r}+A_{1}\right)^{2}-\frac{B^{\prime}}{2 B}\left(\frac{d r}{d \tau}\right)^{2}-\frac{B L^{2}}{r^{3}}=-\frac{G M}{r^{2}}\left(\frac{G M}{c^{2} r}+A_{1}\right)
$$


In fact, we have already found $\frac{d r}{d \tau}$ in (36) through the interval, and it is easy to check that its value is the solution of the equation of motion (38).

Since according to (32) $\frac{d \varphi}{d \tau}=\frac{L}{r^{2}}$, then dividing $\frac{d r}{d \tau}$ from (36) by $\frac{d \varphi}{d \tau}$, we find the equation of motion of the test body near the Sun in the polar coordinates:

$$
\begin{aligned}
& \frac{d r}{d \varphi}= \pm \frac{r^{2}}{L} \sqrt{c^{2}\left(\frac{G M}{c^{2} r}+A_{1}\right)^{2}-\frac{B L^{2}}{r^{2}}-B c^{2}} . \\
& \varphi= \pm L \int \frac{d r}{r^{2} \sqrt{c^{2}\left(\frac{G M}{c^{2} r}+A_{1}\right)^{2}-\frac{B L^{2}}{r^{2}}-B c^{2}}}+A_{2} .
\end{aligned}
$$

The relation (40) is the solution of the problem in the general case. There is a special case in which the initial velocity $V_{0}$ of the test body is zero, or is directed straight to the Sun. In this case the angular momentum of the test body is zero, $L=0$, and the angle of incidence of the test body does not change with time. In other cases, during the motion of the test body, it may, depending on the direction and the magnitude of the initial velocity, get close to the Sun for the minimal distance $R$ and then again move away from the Sun, deflecting at some angle. With the distance $R$ the radial velocity becomes equal to zero: $\dot{r}=\frac{d r}{d t}=0$. At this point, the total velocity of the test body $V$ is perpendicular to the radius-vector directed from the Sun, and is equal to the tangential component of velocity. From (35) with $\dot{r}=0$ taking into account the relations (2) for $B$ we see that the constant $L$ can be found through $R$ and the initial velocity, which is included through $A_{1}$ in (27): 


$$
L=R c \sqrt{\frac{\left(\frac{G M}{c^{2} R}+A_{1}\right)^{2}}{1+\frac{G M \alpha}{R c^{2}}-\frac{\beta G^{2} M^{2}}{R^{2} c^{4}}}}-1 .
$$

We can compare the relativistic solution (40) with the formula for the motion of the particle in gravitational field of the central type in the classical case [17]:

$$
\varphi= \pm L \int \frac{d r}{r^{2} \sqrt{2(\varepsilon-\psi)-\frac{L^{2}}{r^{2}}}}+A_{2}
$$

where $\varepsilon$ is the quantity, which is proportional to the total energy of the particle and at infinity is equal to $\frac{V_{0}^{2}}{2}$,

$$
\psi=-\frac{G M}{r} \text { is the potential of the gravitational field. }
$$

If in (40) we neglect the curvature of spacetime, assuming $B=1$, eliminating the small terms of the form $\frac{\beta G^{2} M^{2}}{c^{4} R^{2}}$ and $\frac{V_{0}^{4}}{c^{4}}$, and if we subtract under the root the rest energy of the unit mass, equal to $c^{2}$, then (40) turns into (42).

\section{Equation of radial motion in GTR}

The standard equations of motion of the test particle near the massive body in the general theory of relativity (GTR) was described, for example, in [18]: 


$$
\frac{d^{2} x^{i}}{d \tau^{2}}+\Gamma_{k m}^{i} \frac{d x^{k}}{d \tau} \frac{d x^{m}}{d \tau}=0
$$

where $\tau$ is the proper time of the moving particle as it determined in GTR.

Since the interval can be expressed through the differential of the proper time of the test particle in the form $d s=c d \tau$, then (16) can be written as follows:

$$
c^{2}=g_{i k} \frac{d x^{i}}{d \tau} \frac{d x^{k}}{d \tau}
$$

The non-zero Christoffel symbols for the spherical coordinates are:

$$
\begin{gathered}
\Gamma_{01}^{0}=\Gamma_{10}^{0}=\frac{B^{\prime}}{2 B}, \quad \Gamma_{00}^{1}=\frac{B^{\prime}}{2 K}, \quad \Gamma_{11}^{1}=\frac{K^{\prime}}{2 K}, \quad \Gamma_{22}^{1}=-\frac{E^{\prime}}{2 K}, \quad \Gamma_{33}^{1}=-\frac{E^{\prime} \sin ^{2} Q}{2 K}, \\
\Gamma_{12}^{2}=\Gamma_{21}^{2}=\Gamma_{13}^{3}=\Gamma_{31}^{3}=\frac{E^{\prime}}{2 E}, \quad \Gamma_{33}^{2}=-\sin Q \cos Q, \quad \Gamma_{23}^{3}=\Gamma_{32}^{3}=\operatorname{ctg} Q .
\end{gathered}
$$

With $i=0$ in (43) and the metric tensor (1) in (45) only two Christoffel symbols are nonzero: $\Gamma_{01}^{0}=\Gamma_{10}^{0}=\frac{B^{\prime}}{2 B}$. Using $x^{0}=c t$ and $x^{1}=r$, taking into account the definition $B^{\prime}=\frac{d B}{d r}$ and multiplying (43) by $\frac{B}{c}$, we find:

$$
B \frac{d^{2} t}{d \tau^{2}}+\frac{d B}{d \tau} \frac{d t}{d \tau}=0, \quad \frac{d}{d \tau}\left(B \frac{d t}{d \tau}\right)=0, \quad B \frac{d t}{d \tau}=A_{3},
$$


where $A_{3}$ is some constant, which can be conveniently associated with the initial velocity at infinity. Indeed, at infinity $B=1$, and the coordinate time $t$ is the time of the inertial reference frame in which the particle is moving at the constant velocity $V_{0}$. Then, according to the special theory of relativity, $d \tau=d t \sqrt{1-V_{0}^{2} / c^{2}}$ and $A_{3}=\frac{1}{\sqrt{1-V_{0}^{2} / c^{2}}}$.

For the motion of the particle along the radius the angular coordinates $Q$ and $\varphi$ do not change, and $d Q=d \varphi=0$. In this case, from (44) for the coordinates $x^{0}=c t$ and $x^{1}=r$ taking into account (1) and $d \tau$ from (46) we obtain:

$$
c^{2}=\frac{A_{3}^{2} c^{2}}{B}-\frac{A_{3}^{2} K}{B^{2}}\left(\frac{d r}{d t}\right)^{2}
$$

According to the Schwarzschild metric $B_{o}=B=g_{00}=\frac{1}{K}=1-\frac{2 G M}{r c^{2}}$, so with $A_{3}=\frac{1}{A_{4}}$ for radial motion we have:

$$
\frac{d r}{d t}= \pm B_{o} c \sqrt{1-A_{4}^{2} B_{o}}= \pm c\left(1-\frac{2 G M}{r c^{2}}\right) \sqrt{1-A_{4}^{2}\left(1-\frac{2 G M}{r c^{2}}\right)}
$$

In a more general case, going over from the spherical coordinates to the Cartesian coordinates and then to the polar coordinates, as in the previous section, the equations (43) can be reduced to the following form:

$$
\frac{d r}{d \tau}= \pm \sqrt{c^{2} A_{3}^{2}-\frac{B_{o} L^{2}}{r^{2}}-B_{o} c^{2}}
$$




$$
\frac{d r}{d t}= \pm \frac{B_{o}}{A_{3}} \sqrt{c^{2} A_{3}^{2}-\frac{B_{o} L^{2}}{r^{2}}-B_{o} c^{2}}, \quad \frac{d r}{d \varphi}= \pm \frac{r^{2}}{L} \sqrt{c^{2} A_{3}^{2}-\frac{B_{o} L^{2}}{r^{2}}-B_{o} c^{2}}
$$

These equations are used in GTR to describe the planar motion of the bodies relative to the fixed center in the polar coordinates.

\section{Pioneer anomaly}

\subsection{Qualitative approach}

We shall assume that the spacecraft moves away from Earth and the Sun almost radially, transmitting to the tracking station the radio signal of the known frequency $v_{0}$. Due to the Doppler effect, the frequency received on Earth will change to the value:

$$
v=\frac{v_{0} \sqrt{1-V^{2} / c^{2}}}{1-V / c \cos \theta} \approx v_{0}\left(1-\frac{V}{c}\right)
$$

where $V$ is the velocity of the spacecraft relative to the Earth,

$\theta \approx \pi$ is the angle between the velocity and the direction to the radiation detector.

As the spacecraft is getting farther from the Sun with the turned off engines under the influence of solar attraction the velocity $V$ gradually decreases, so that the frequency $v$ should increase. From (49) we can obtain the change of the velocity of the spacecraft and the relative change of the frequency during the time $\Delta t$ in which the signal goes from the spacecraft to the Earth: 


$$
\frac{\Delta v}{v_{0}}=-\frac{\Delta V}{c}=-\frac{a_{f} \Delta t}{c}
$$

where $a_{f}$ is the total acceleration of the spacecraft.

The acceleration $a_{f}$ is negative, because it is mostly caused by the Sun and directed towards the Sun, and the velocity $V$ is directed at the angle $\approx \pi$ away from the direction from the spacecraft to the Sun. We shall further assume that the relative change in the frequency of the signal (50) is of such kind that it takes into account all the possible sources of acceleration and the factors influencing the result. Then the residual signal, which is not simulated by anything, can also be represented by the formula (50), in which in the place of acceleration the anomalous acceleration $a_{p}$ stands:

$$
\left(\frac{\Delta v}{v_{0}}\right)_{p}=-\frac{a_{p} \Delta t}{c} .
$$

We can estimate the velocity of the spacecraft depending on the radial distance $r$ from the equation of its free radial motion in the classical mechanics:

$$
\frac{d^{2} r}{d t^{2}}=\frac{1}{2} \frac{d}{d r}\left(\frac{d r}{d t}\right)^{2}=-\frac{G M}{r^{2}},
$$

where $M$ is the Sun's mass. 
Assuming in the first approximation the motion of the spacecraft purely radial, we shall integrate this equation: $\frac{d r}{d t}=V=\sqrt{\frac{2 G M}{r}+A_{5}}$. We shall assume the velocity of the spacecraft at the distance of 87 a.u. was $12.2 \mathrm{~km} / \mathrm{s}$, from this we find $A_{5}=1.28 \cdot 10^{8} \mathrm{~m}^{2} / \mathrm{c}^{2}$. Consequently, at the distance $R=20$ a.u. for the velocity of the spacecraft in the approximation of the free radial motion we should assume about $14.7 \mathrm{~km} / \mathrm{s}$.

We can explain the effect of Pioneers in the following way. From (35) in the approximation of the radial motion, when the density of the angular momentum $L=0$, and with $B_{k}=B=g_{00}=1+\frac{G M \alpha}{r c^{2}}-\frac{\beta G^{2} M^{2}}{r^{2} c^{4}} \approx 1$, for the radial velocity of freely flying spacecraft in CTG we can write down:

$$
\frac{d r}{d t}=c \sqrt{1-\frac{1}{\left(\frac{G M}{c^{2} r}+A_{1}\right)^{2}}} .
$$

If we proceed from (52), at the distance of 1 a.u. we can assume that the initial velocity is equal to $V_{1}=4.361 \cdot 10^{4} \mathrm{~m} / \mathrm{s}$. This allows us to estimate in (53) the value of the constant $A_{1}=1.00000000071$ and to find the velocity of the spacecraft at different distances.

In GTR we have a similar formula according to (47):

$$
\frac{d r}{d t}=c\left(1-\frac{2 G M}{r c^{2}}\right) \sqrt{1-A_{4}^{2}\left(1-\frac{2 G M}{r c^{2}}\right)}
$$


Substituting in (54) $\frac{d r}{d t}=V_{1}=4.361 \cdot 10^{4} \quad \mathrm{~m} / \mathrm{s} \quad$ with $\quad r=1 \quad$ a.u., we find $A_{4}^{2}=0.999999998579$. With the help of (53) and (54) we calculate the velocity of the spacecraft according to CTG and GTR at different distances for the case of conditionally radial motion. The results are shown in Table 1.

As we can see, the velocity of the spacecraft in GTR and CTG are slightly different. If the spacecraft starts with $r=1$ a.u., then the time of its motion up to $r=5$ a.u. is of the order of $\Delta t_{1-5}=1.82 \cdot 10^{7} \mathrm{~s}$ (this approximate value is obtained by dividing the distance traveled by the average velocity). During this time up to the position with $r=5$ a.u. due to the different velocities the difference between the positions of the spacecrafts according to the equations of GTR and CTG will grow up to $\Delta r_{1-5}=3.29 \cdot 10^{5} \mathrm{~m}$. For the spacecraft to move from 5 to 10 a.u. the time is required accordingly about $\Delta t_{5-10}=3.79 \cdot 10^{7} \mathrm{~s}$, what is shown in Table 1 .

Table 1. The data on the motion of the spacecraft

\begin{tabular}{|c|c|c|c|c|}
\hline$r$, a.u. & $V, 10^{4} \mathrm{~m} / \mathrm{s}$ & $\Delta r, 10^{5} \mathrm{~m}$ & $\Delta t, 10^{7} \mathrm{~s}$ & $a_{p}, 10^{-10} \mathrm{~m} / \mathrm{s}^{2}$ \\
\hline 1 & 4.361 & & & \\
\hline 5 & $2.196624147 \mathrm{CTG}$ & 3.29 & 1.82 & 19.8 \\
\hline 10 & $\begin{array}{c}1.746714927 \mathrm{CTG} \\
1.746712488 \mathrm{GTR}\end{array}$ & 13.2 & 3.79 & 18.3 \\
\hline 15 & $1.568321059 \mathrm{CTG}$ & 9.47 & 4.51 & 9.31 \\
\hline 20 & $1.568319299 \mathrm{GTR}$ & & & \\
\hline & $1.471033615 \mathrm{CTG}$ & \multirow{2}{*}{7.69} & 4.92 & 6.4 \\
\hline
\end{tabular}


Since the velocity of the spacecraft in CTG is somewhat greater than in GTR, then in case of the measurements according to the Doppler effect at each time point the spacecraft is located farther than it is assumed according to GTR. Due to this difference in the distances the velocity of the spacecraft, always decreasing with time due to the attraction of the Sun, is less than the velocity of the spacecraft according to GTR. For example, at the distance $r+\Delta r_{1-5}=$ 5 a.e. $+3.29 \cdot 10^{5} \mathrm{~m}$ according to CTG the velocity of the spacecraft in our model calculations will be $2.196478495 \cdot 10^{4} \mathrm{~m} / \mathrm{s}$, whereas according to GTR the spacecraft is at the distance of 5 a.u. and has the velocity $2.196620538 \cdot 10^{4} \mathrm{~m} / \mathrm{s}$. As a result, with the help of the Doppler effect, the velocity of the spacecraft is registered, decreased relative to the data of GTR. This decrease is attributed to the anomalous acceleration acting in the direction towards the Sun.

In the last column of Table 1, we estimated the anomalous acceleration by the formula: $a_{p}=\frac{2 \Delta r}{(\Delta t)^{2}}$. This acceleration means that the spacecraft is as though at the distance smaller by the value $\Delta r$, that arises during the time $\Delta t$ due to the difference in velocities. The distances $\Delta r$ in Table 1 are calculated by an average velocity at each interval of motion, so to obtain the total result we should add up all $\Delta r$. This will lead over time to the increase in distance between the positions of the spacecrafts according to GTR and CTG, and to decrease of the anomalous acceleration $a_{p}$ with the distance as compared with the data in Table 1. As it is shown in Table 1, the values of the anomalous acceleration are close enough to the data obtained for the effect of Pioneers, and at small distances up to 5-10 a.u. they are masked by the acceleration from the pressure force of the solar wind.

\subsection{Analytical approach}

Now let us try to derive the corresponding formula for the anomalous acceleration, again for the case of purely radial motion. Assuming in (35) $L=0$, for the velocity of the spacecraft in CTG and for its current position relative to the Sun we obtain: 


$$
\begin{gathered}
\frac{d r}{d t}=B c \sqrt{1-\frac{B}{\left(\frac{G M}{c^{2} r}+A_{1}\right)^{2}}}, \\
t+A_{6}=\frac{A_{1}}{B c\left(A_{1}^{2}-B\right)} \sqrt{\frac{G^{2} M^{2}}{c^{4}}+\frac{2 A_{1} G M r}{c^{2}}+\left(A_{1}^{2}-B\right) r^{2}}- \\
-\frac{G M}{c^{3}\left(A_{1}^{2}-B\right)^{3 / 2}} \ln \left(\sqrt{A_{1}^{2}-B} \sqrt{\frac{G^{2} M^{2}}{c^{4}}+\frac{2 A_{1} G M r}{c^{2}}+\left(A_{1}^{2}-B\right) r^{2}}+\left(A_{1}^{2}-B\right) r+\frac{A_{1} G M}{c^{2}}\right),
\end{gathered}
$$

where $B=g_{00}=1+\frac{G M \alpha}{r c^{2}}-\frac{\beta G^{2} M^{2}}{r^{2} c^{4}} \approx 1$ is the time component of the metric in CTG, $A_{1}=\left(\frac{d t}{d \tau}\right)_{\infty}=\frac{1}{\sqrt{1-V_{0}^{2} / c^{2}}}$, where $V_{0}$ is the velocity of the spacecraft at infinity, the radial coordinate $r$ is the function of the time $t$ of the motion from the Sun, and the constant $A_{6}$ is the parameter of integration.

If at a given time point $t=t_{0}$ we know the radial distance $r=r_{0}$ and the velocity $\frac{d r}{d t}=V_{0}$, it allows us to calculate the constants $A_{1}$ and $A_{6}$ in (55) and (56). Thus, in the previous section, we assumed for simplicity that $B \approx 1$, at $t=0$ the spacecraft was at a distance $r=1$ a.u., and the constant $A_{1}=1.00000000071$. These data can be used to estimate the constant $A_{6}$ in (56).

We will integrate now the equation (54) for the radial motion in GTR: 


$$
\begin{aligned}
& t+A_{7}=\frac{r \sqrt{1-A_{4}^{2}\left(1-\frac{2 G M}{r c^{2}}\right)}}{c\left(1-A_{4}^{2}\right)}-\frac{2 G M}{c^{3}} \ln \frac{1+\sqrt{1-A_{4}^{2}\left(1-\frac{2 G M}{r c^{2}}\right)}}{1-\sqrt{1-A_{4}^{2}\left(1-\frac{2 G M}{r c^{2}}\right)}}+ \\
& +\frac{\left(3 A_{4}^{2}-2\right) G M}{c^{3}\left(1-A_{4}^{2}\right) \sqrt{1-A_{4}^{2}}} \ln \frac{\sqrt{1-A_{4}^{2}\left(1-\frac{2 G M}{r c^{2}}\right)}-\sqrt{1-A_{4}^{2}}}{\sqrt{1-A_{4}^{2}\left(1-\frac{2 G M}{r c^{2}}\right)}+\sqrt{1-A_{4}^{2}}} .
\end{aligned}
$$

In (57) the constant $A_{7}$ appears, which must be found together with the constant $A_{4}$ from the initial conditions of motion.

We suppose now that we have derived from (56) the dependence of the radial distance in CTG as the function of time: $r(t)=r_{k}(t)$. Similarly, from (57) we can determine the dependence of the radial distance in GTR as the function of time: $r(t)=r_{o}(t)$. At a first approximation, the gravitational acceleration of the Sun depends on the radial distance according to the Newton's formula, and we can write for the accelerations in CTG and GTR the following:

$$
g_{k}(t)=-\frac{G M}{r_{k}^{2}(t)}, \quad \quad g_{o}(t)=-\frac{G M}{r_{o}^{2}(t)}
$$

The anomalous acceleration as the function of the time of the spacecraft's radial motion is found as the difference between these accelerations:

$$
a_{p}(t)=g_{o}(t)-g_{k}(t)=-\frac{G M\left[r_{k}^{2}(t)-r_{o}^{2}(t)\right]}{r_{k}^{2}(t) r_{o}^{2}(t)}
$$


The meaning of this equality is that in case of Pioneers the acceleration $g_{o}(t)$, calculated in GTR, is overrated in the absolute value as compared to the measured acceleration. If the acceleration $g_{k}(t)$ in CTG describes the motion more precisely and is equal to the measured acceleration, then in order to obtain it we should subtract the anomalous acceleration from the acceleration in GTR: $g_{k}(t)=g_{o}(t)-a_{p}(t)$.

\section{Conclusion}

In general theory relativity (GTR) the gravitational field is the same as the metric field with its metric tensor. As a result the gravitational field does not create the metric similar to electromagnetic field in equation for the metric, and the metric tensor is calibrated with the help of Newton's law of universal gravitation. We can suppose that such calibration is not accurate since the Newton's law has no relativistic corrections. On the other hand in the covariant theory of gravitation (CTG) the gravitational field is a fundamental field which has its stress-energy tensor and can influence the metric in equation for the metric. The metric component $B_{k}=g_{00}=1+\frac{G M \alpha}{r c^{2}}-\frac{\beta G^{2} M^{2}}{r^{2} c^{4}}$ in CTG depends on the energy of gravitational field and it seems is more precise then $B_{o}=g_{00}=\frac{1}{K}=1-\frac{2 G M}{r c^{2}}$ in GTR. The metric component $g_{00}$ is in both equations of motion in CTG and GTR but the equations are different.

From the point of view of CTG the effect of Pioneers is explained as the result of using equation of motion which is does not coincide with the equation of motion of GTR.

All computer calculations associated with the motion of the spacecrafts obligatorily use GTR and take into account not only the influence of the Sun, but of other planets. If the equation of motion of $\mathrm{CTG}$ is valid, in the effect of Pioneer there is no anomalous acceleration, and the effect is due to the use of GTR instead of CTG. The following fact also 
points out to the probable inaccuracy of GTR that in the signal from Pioneers we could see not simulated periodic changes associated with the diurnal rotation of the Earth and its annual revolution around the Sun. From Table 1 it follows also that the velocities of the spacecraft in GTR and CTG at equal distances can differ by several $\mathrm{cm} / \mathrm{s}$. At the same time, in several articles the so-called flyby effect has been described, when the velocity of spacecrafts differs from the calculated values up to several $\mathrm{cm} / \mathrm{s}$ [19-20].

There are also works such as [21] - [23], according to which the motion of the comets: Halley's comet, Encke and others, after their passing near the planets disturbances of unknown nature are discovered, which are not taken into account by GTR equations (48). We can assume that the recalculation of the motion of spacecrafts and comets in terms of CTG with the help of equations (35), (36), (40) will improve the situation.

\section{References}

1. J.D. Anderson, P.A. Laing, E.L. Lau, A.S. Liu, M.M. Nieto and S.G. Turyshev. Phys. Rev. D 65, 082004 (2002). doi:10.1103/PhysRevD.65.082004.

2. S.G. Turyshev, V.T. Toth, G. Kinsella, Siu-Chun Lee, S.M. Lok, and J. Ellis. Phys. Rev. Lett. 108, 241101 (2012). doi:10.1103/PhysRevLett.108.241101.

3. D. Modenini and P. Tortora. Phys. Rev. D 90, 022004 (2014). doi:10.1103/PhysRevD.90.022004.

4. G.U. Varieschi. Phys. Res. Int. 2012, 469095 (2012). doi:10.1155/2012/469095.

5. A.F. Rañada and A. Tiemblo. Can. J. Phys. 90, 931 (2012). doi:10.1139/p2012-086.

6. M.W. Kalinowski. CEAS Space J. 5, 19 (2013). doi:10.1007/s12567-013-0042-9.

7. J.D. Anderson and J.R. Morris. Phys. Rev. D 86, 064023 (2012). doi:10.1103/PhysRevD.86.064023.

8. G.S.M. Moore and R.E.M. Moore. Astrophys. Space. Sci. 347, 235 (2013). doi:10.1007/s10509-013-1514-2. 
9. P.C. Ferreira. Adv. Space Res. 51, 1266 (2013). doi:10.1016/j.asr.2012.11.004.

10. M.R. Feldman. PLoS ONE. 8, e78114 (2013). doi:10.1371/journal.pone.0078114.

11. S.G. Fedosin. Fizicheskie teorii i beskonechnaia vlozhennost' materii. Perm. 2009.

12. S.G. Fedosin. Int. Front. Sci. Lett. 1, 48 (2014).

13. W. Pauli. Theory of Relativity. Dover Publications, New York. 1981.

$\begin{array}{lllllll}\text { 14. S.G. Fedosin. } & \text { Adv. } & \text { Nat. } & \text { Sci. } & \text { 5, } & 55 & \text { (2012). }\end{array}$ doi:10.3968\%2Fj.ans.1715787020120504.2023.

15. S.G. Fedosin. Int. J. Thermo. 18, 13 (2015). doi:10.5541/ijot.34003.

16. S.G. Fedosin. The concept of the general force vector field. vixra.org, 28 June 2014. http://vixra.org/abs/1406.0173.

17. L.D. Landau and E.M. Lifshitz. Mechanics, Vol. 1, 3th ed. Butterworth-Heinemann. 1976.

18. L.D. Landau and E.M. Lifshitz. The Classical Theory of Fields, Vol. 2, 4th ed. Butterworth-Heinemann. 1975.

19. J.D. Anderson and J.G. Williams. Class. Quantum Gravity. 18, 2447 (2001). doi:10.1088/0264-9381/18/13/307.

20. J.D. Anderson, J.K. Campbell and M.M. Nieto. New Astron. 12, 383 (2007). doi:10.1016/j.newast.2006.11.004.

21. F.L. Whipple. Astrophys. J. 111, 375 (1950). doi:10.1086/145272.

22. B.G. Marsden. Planet. Space Sci. 57, 1098 (2009). doi:10.1016/j.pss.2008.12.007.

23. T. Kiang. Mon. Not. R. Astron. Soc. 162, 271. (1973). doi:10.1093/mnras/162.3.271. 